\section{The human touch in lung injury}

\section{By Kai-Jye Lou, Staff Writer}

Despite improvements in critical care and mechanical ventilation protocols, the mortality rate for patients with acute lung injury still hovers at $30 \%-40 \% .{ }^{1-3}$ A group at the University of California, San Francisco thinks allogeneic bone marrow-derived mesenchymal stem cells could lower mortality and has shown the therapeutic effect of these cells in a model of acute lung injury that uses lungs from human cadavers perfused by an artificial circulatory system. ${ }^{4}$

The results also could bolster the case for using a growth factor produced by the mesenchymal stem cells (MSCs) to treat the condition.

"This work is the first demonstration that human mesenchymal stem cells can reverse injury in the human lung," said Michael Matthay, a professor of medicine, anesthesia and critical care at UCSF and a senior research associate at the university's Cardiovascular Research Institute. "We have previously depended on mouse models, but now we are testing therapies using human cells in the human lung-and we found that these cells can reverse acute lung injury."

Acute lung injury and its more severe manifestation, acute respiratory distress syndrome (ARDS), are inflammatory disorders most commonly caused by sepsis, pneumonia, trauma or the aspiration of damaging substances and objects. Treatment is limited to supportive measures that assist in recovery, such as mechanical ventilation, and utilizing damaged but still functional lung areas.

"Basically, there is no clinical therapy available to patients with acute lung injury," said Mauricio Rojas, an assistant professor in the Division of Pulmonary, Allergy and Critical Care Medicine at the Emory University School of Medicine. "Patients are placed on mechanical ventilation, but there is nothing out there that says you could give this compound and the patient will get better."

Earlier mouse studies from the UCSF group and others have suggested that MSCs could improve outcomes in acute lung injury. ${ }^{5-7}$ However, the translational track record for treatments has been disappointing.

Daniel Weiss, an associate professor of medicine at The University of Vermont College of Medicine, said data from recent studies evaluating MSCs in mouse models of acute lung injury have been compelling. However, he added that a lot of work evaluating other treatments in mouse models has not translated to humans.

As an example, Weiss noted that anti-tumor necrosis factor (TNF) treatments protected mice from acute lung injury but did not have significant efficacy in humans. Other approaches that showed efficacy in animal models, such as IL-1 inhibitors and surfactant-based treatments, have also failed in the clinic. ${ }^{8}$

To bridge the translational gap, the UCSF research turned to an $e x$ vivo model that used perfused lungs isolated from human cadavers. ${ }^{9}$ This model, published in 2007, allowed the researchers to study alveolar fluid clearance in a human system under conditions that resemble pulmonary edema. Edema is a key component of acute lung injury pathophysiology.

In the new study, the UCSF researchers used their model to evaluate the therapeutic effect of MSCs. Treatment of human lung preparations with allogeneic human MSCs one hour after endotoxin-induced injury restored alveolar fluid clearance and reduced signs of pulmonary edema to levels comparable with those in uninjured controls.

Treatment with MSC-conditioned medium produced a similar effect. Moreover, the group found that keratinocyte growth factor (KGF) - which is produced by MSCs-was responsible for about $80 \%$ of the protective effects seen with the medium.

The findings were published in the Proceedings of the National Academy of Sciences.

"We were very concerned that the results observed in animal models would not translate to humans, but the data from the PNAS study suggests that it will," Rojas told SciBX. "The work is opening the door for the use of a cell-based therapy in acute lung injury."

Luis Ortiz, director of the Division of Occupational Medicine and an associate professor in the Department of Environmental and Occupational Health at the University of Pittsburgh, noted that the ex vivo perfused human lung model cannot fully recapture the inflammatory processes associated with acute lung injury. "However, even with this limitation, you could still acknowledge that human mesenchymal stem cells can alleviate the signs of injury in the human lung," he said.

Matthay, who is the senior author on the PNAS paper, agreed that the model cannot recapitulate some aspects of acute lung injury-for example it does not reflect the influence from other organs like the liver or spleen, which could mount significant inflammatory responses during acute lung injury.

Nevertheless, Weiss thinks the model's benefits far outweigh that shortcoming. "The work is very exciting and represents a critical step in moving a therapy based on human mesenchymal stem cells toward clinical trials in acute lung injury and acute respiratory distress syndrome," he said.

\section{Another factor to consider}

Jae Lee, lead author on the PNAS paper and an associate professor in the Department of Anesthesiology at UCSF, said more steps are needed before moving into clinical trials evaluating MSCs for acute lung injury. "We want to elucidate the mechanisms before translating this therapy to the clinic," he said.

Nailing down the mechanism is important because it's unclear whether it would be better to treat patients with MSCs or just the relevant factors produced by the cells, such as KGF.

Indeed, recombinant proteins could be an attractive alternative to 
MSCs because some of the legwork has already been done.

Kepivance palifermin, a recombinant human $\mathrm{KGF}$, is marketed by Biovitrum $\mathbf{A B}$ to treat severe oral mucositis. Moreover, the biologic's original developer, Amgen Inc., has shown that i.v. recombinant human KGF protected mice and rats from pulmonary injury. ${ }^{10}$

"The paper is important as it shows that $\mathrm{KGF}$ - and not necessarily stem cells-are primarily contributing to the therapeutic effects seen in this human model of acute lung injury," said Björn Skölving, medical information manager for Kepivance at Biovitrum. "However, the work is still preclinical, so we need to remain cautious on any implications before clinical studies are carried out."

Hans Siltberg, director of scientific communications at Biovitrum, said that at this time the primary focus with Kepivance is on developing the product's clinical side.

Although the results in the PNAS paper showed that KGF improved one of the symptoms central to acute lung injury-pulmonary edema-both Rojas and Weiss said the other pathways involved in acute lung injury also need to be addressed.

Weiss noted that MSCs secrete a broad range of mediators, including those that modulate the inflammatory response. "The cells could potentially affect the multiple pathways involved in acute lung injury," he said.

"KGF is an important element in decreasing severity of acute lung injury by restoring the integrity of the alveolar space, but it is not the only one," said Rojas. "What we need to remember is that acute lung injury is a systemic entity and it's not only a matter of handling fluid accumulation in the lungs."

Pittsburgh's Ortiz thinks that the positive effects of KGF reflect the limitations of the ex vivo model, which does not fully capture the inflammatory component of acute lung injury. He thinks candidate therapies also will need to address the inflammatory aspects.

Matthay thinks it is still too early to know whether human MSCs, an MSC-conditioned medium or KGF alone would make the best treat- ment. "At this point, we have to equipoise on whether to give the cells or the factors directly. I think mesenchymal stem cells are still the focus, but it is conceivable that our future work may suggest that giving factor alone is the way to go," he said.

MSCs could have a delivery advantage over growth factors alone. Matthay noted that systemically administered MSCs migrate to sites of injury.

Lee and Matthay said their group is now fleshing out the mechanisms of action of human MSCs in models of acute lung injury and is exploring the design of a clinical trial. "Realistically, we are looking at Phase I trials focused initially on safety in about one to two years," Lee told SciBX.

The patent and licensing status of the work described in the PNAS paper is undisclosed.

Lou, K.-J. SciBX 2(37); doi:10.1038/scibx.2009.1394

Published online Sept. 24, 2009

\section{REFERENCES}

1. Zambon, M. \& Vincent, J.-L. Chest 133, 1120-1127 (2008)

2. Erickson, S.E. et al. Crit. Care Med. 37, 1574-1579 (2009)

3. Rubenfeld, G.D. et al. N. Engl. J. Med. 353, 1685-1693 (2005)

4. Lee, J.W. et al. Proc. Natl. Acad. Sci. USA; published online Aug. 31, 2009; doi:10.1073/pnas.0907996106

Contact: Jae W. Lee, University of California, San Francisco, Calif. e-mail: leejw@anesthesia.ucsf.edu

5. Gupta, N. et al. J. Immunol. 179, 1855-1863 (2007)

6. Rojas, M. et al. Am. J. Respir. Cell Mol. Biol. 33, 145-152 (2005)

7. Ortiz, L.A. et al. Proc. Natl. Acad. Sci. USA 100, 8407-8411 (2003)

8. Reyal, Y. \& Bellingan, G. Surgery 22, III-VII (2004)

9. Frank, J.A. et al. Am. J. Physiol. Lung Cell. Mol. Physiol. 293, L52-L59 (2007)

10. Guo, J. et al. Am. J. Physiol. Lung Cell. Mol. Physiol. 275, L800-L805 (1998)

\section{COMPANIES AND INSTITUTIONS MENTIONED}

Amgen Inc. (NASDAQ:AMGN), Thousand Oaks, Calif.

Biovitrum AB (SSE:BVT), Stockholm, Sweden

Emory University School of Medicine, Atlanta, Ga.

University of California, San Francisco, Calif.

University of Pittsburgh, Pittsburgh, Pa.

The University of Vermont College of Medicine, Burlington, Vt. 